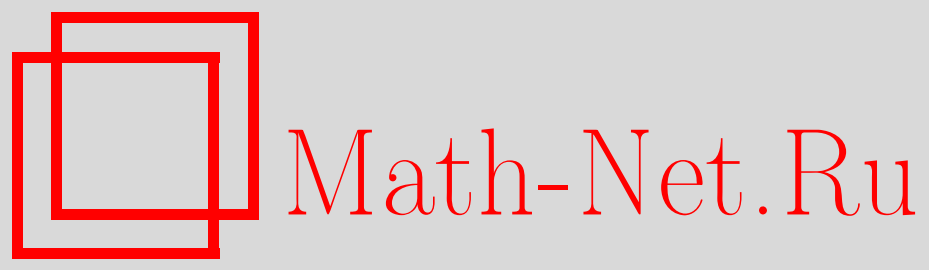

В. М. Мануйлов, О почти коммутирующих операторах, Функи. анализ и его прил., 1997, том 31, выпуск 3, 80-82

DOI: https://doi.org/10.4213/faa482

Использование Общероссийского математического портала Math$\mathrm{Net.Ru}$ подразумевает, что вы прочитали и согласны с пользовательским соглашением

http://www . mathnet.ru/rus/agreement

Параметры загрузки:

IP : 54.162 .27 .143

26 апреля 2023 г., 13:47:45

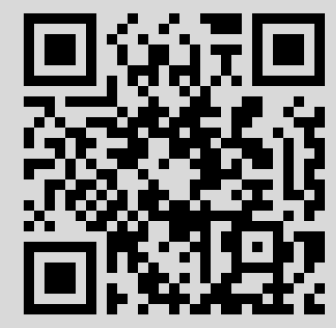


(1995). 8. Степин C. A. Функц. анализ и его прил., 29, вып. 2, $22-35$ (1995). 9. Mourre E. Comm. Math. Phys., 78, 391-408 (1981). 10. Amrein W., Boutet de Monvel A., Georgescu V. $C_{0}$-Groups, Commutator Methods and Spectral Theory of $N$-Body Hamiltonians. Birkhäuser, Basel (1996). 11. Цикон Х., Фрезе Р., Кири В., Саймон Б. Операторы Шрёдингера с приложениями к квантовой механике и глобальной геометрии. Мир, М. (1990). 12. Ландау Л. Д., Лифиии Е. М. Гидромеханика. Наука, М. (1986). 13. Lifschitz A. E. Magnetohydrodinamics and spectral theory. Kluwer Acad. Publishers, Dodrecht (1989). 14. Копачевский Н. Д., Крейн С. Г., Нго Зуй Кан. Операторные методы в линейной гидродинамике. Наука, М. (1989).

Киевский университет,

кафедра математического анализа

Поступило в редакцию 28 июля 1996 г.

УДК 517.98

\section{О почти коммутирующих операторах *}

(c) 1997. В. М. МАнУйлов

В настоящей заметке исследуется вопрос о возможности прогомотопировать один из двух почти коммутирующих операторов к единице так, чтобы норма коммутатора вдоль всего пути была мала. В общем случае ответ отрицательный $[4,2]$, более того, в работе [2] определено топологическое препятствие для существования такой гомотопии. Мы приводим случай, когда такая гомотопия существует. Напомним, что условие $R R(A)=0$ означает [1], что среди самосопряженных элементов $C^{*}$-алгебры $A$ плотны элементы с конечным спектром; поэтому без ограничения общности можно считать, что $h=\sum_{i=1}^{n} \lambda_{i} p_{i}-$ конечная линейная комбинация проекторов $p_{i} \in A$, где собственные значения упорядочены по убыванию. Напомним также, что условие $\operatorname{tsr}(A)=1$ означает [3], что обратимые элементы плотны в $A$.

Tеорема 1. Пусть $A$ есть $C^{*}$-алгебра со следуюшими свойствами:

(i) $R R(A)=0 \quad u \operatorname{tsr}(A)=1$;

(ii) для любого проектора $p \in A$ унитарная группа $C^{*}$-алгебры $p A p$ свлзна. Пусть $h, u \in A-$ самосопряженный и унитарньй элементы, такие, что

$$
\left\|u^{*} h u-h\right\|<\delta .
$$

Тогда существует константа $C$, зависящал только от $\|h\|$, и путь $u(t)$, соединяющий и с 1 , такой, что при достаточно малом $\delta$ для всех $t$

$$
\left\|u^{*}(t) h u(t)-h\right\|<C \sqrt[4]{\delta} .
$$

ДоказАтельство. Разобьем спектр оператора $h$ на мелкие отрезки длины $\sqrt[4]{\delta}$ и пронумеруем те из них, которые содержат хотя бы одну точку спектра. Тогда $\operatorname{Sp}(h) \subset \bigcup_{k=1}^{m} \Delta_{k}$. Без ограничения общности можно считать, что точки спектра оператора $h$ не совпадают с концами отрезков $\Delta_{k}$. Отметим, что если отрезки $\Delta_{k}$ и $\Delta_{k+1}$ не примыкают друг к другу, то спектр оператора $h$ имеет лакуну длины не менее чем $\sqrt[4]{\delta}$. Обозначим спектральные проекторы оператора $h$, отвечающие отрезкам $\Delta_{k}$, через $q_{k}, q_{1}+\cdots+q_{m}=1$.

* Настоящая работа выполнена при частичной поддержке РФФИ (грант №96-01-00182). 
Тогда можно разложить $A$ в прямую сумму, соответствующую этим проектоpaм, $A=\bigoplus_{k=1}^{m} q_{k} A$, и представлять элементы алгебры $A$ как матрицы по отношению к этому разложению, $a=\left(a_{i j}\right)$, где $a \in A, a_{i j}=q_{i} a q_{j}$. Пусть $\mu_{k}$ - середины отрезков $\Delta_{k}$. Определим блочно-трехдиагональную матрицу $d(a)$ следующим образом:

(i) если $j \geqslant i+2$ или $j \leq i-2$, то положим $d(a)_{i j}=0$;

(ii) если $j=i \pm 1$ и $\left|\mu_{j}-\mu_{i}\right|>\sqrt[4]{\delta}$, то положим также $d(a)_{i j}=0$;

(iii) в остальных случаях положим $d(a)_{i j}=a_{i j}$.

Обозначим через $d_{k}(a)$ диагональ матрицы $a$, лежащую на $k$ диагоналей выше (или ниже, если $k$ отрицательно) главной. Неравенство (1) означает, что матрица $u$ почти трехдиагональная:

Лемма 2. Для достаточно мальх $\delta$ выполнено неравенство $\|u-d(u)\|<$ $4\|h\| \sqrt[4]{\delta}$.

Первый шаг гомотопии состоит в том, что мы соединяем матрицы $u$ и $d(u)$ отрезком. Конечно, этот путь не является унитарным, но лежит вблизи унитарной группы $U$ :

$$
\operatorname{dist}(u(t), U)<4\|h\|^{1 / 2} \sqrt[4]{\delta},
$$

и вдоль этого пути $\|u(t) h-h u(t)\|<2 \delta+2\|h\| 4\|h\|^{1 / 2} \sqrt[4]{\delta}$.

Следующая лемма показывает, что можно прогомотопировать матрицу к почти верхнетреугольному виду.

Лемма 3. Пусть $a=\left(\begin{array}{ll}a_{11} & a_{12} \\ a_{21} & a_{22}\end{array}\right)$ - матрица в $q_{j_{1}} A \oplus q_{j_{2}}$ A. Тогда для любого $\varepsilon>0$ существует унитарньй путь $v(t)$, такой, что $v(0)=1 u$ $v(1) a=\left(\begin{array}{ll}a_{11}^{\prime} & a_{12}^{\prime} \\ a_{21}^{\prime} & a_{22}^{\prime}\end{array}\right)$, где $\left\|a_{21}^{\prime}\right\|<\varepsilon$.

Второй шаг гомотопии. Рассмотрим элемент $u_{21}$ матрицы $u$. Если отрезки $\Delta_{1}$ и $\Delta_{2}$ соприкасаются, то элемент $u_{21}$ достаточно мал и по определению $d(u)_{21}=d(u)_{12}=0$. В этом случае положим $v_{1}=1$. В противном случае воспользуемся предыдущей леммой и найдем унитарную $2 \times 2$-матрицу $v_{1}$, умножение на которую уменьшит норму элемента $u_{21}$ почти до нуля. Следующий участок гомотопии определим как путь, определенный умножением $d(u)$ на $v_{1}(t)$ с последующим обнулением элемента $u_{21}^{\prime}$. Затем обратимся к элементу $u_{32}$ и аналогично определим матрицу $v_{2}$, отличную от единичной матрицы только на пересечении 2-й и 3-й строк и столбцов, после умножения на которую можно считать, что $u_{32}^{\prime}=0$. Повторяя эту процедуру, получим путь, определенный унитарной матрицей $v=v_{m-1} \cdots v_{2} v_{1}$. Обозначим $v \cdot d(u)$ через $\bar{u}$. Очевидно, что матрица $\bar{u}$ является трехдиагональной и верхнетреугольной. При этом вдоль указанного пути сохраняется оценка (2). Но тогда из близости матрицы $\bar{u}$ к унитарным норма ее верхнетреугольной части также мала; следовательно, матрица $\bar{u}$ почти диагональна, а именно

$$
\left\|\bar{u}-d_{0}(\bar{u})\right\|<24\|h\|^{1 / 2} \sqrt[4]{\delta},
$$

и, значит, ее диагональные элементы обратимы. Кроме того, поскольку путь, соединяющий $d(u)$ с $\bar{u}$, лежит в множестве четырехдиагональных матриц (диагонали от -1-й до 2-й), норма коммутатора может быть оценена:

$$
\|\overline{\bar{u}}(t) h-h \overline{\bar{u}}(t)\|<6\|h\|^{1 / 2} \sqrt[4]{\delta} .
$$


Третий шаг гомотопии. Соединим матрицы $\bar{u}$ и $d_{0}(\bar{u})$ отрезком. При этом из $(3)$ следует, что $\operatorname{dist}(u(t), U)<28\|h\|^{1 / 2} \sqrt[4]{\delta}$. После того как мы привели матрицу $u$ к блочно-диагональному виду $d_{0}(\bar{u})$, любой путь, лежащий в множестве матрищ такого вида, дает малую норму коммутатора: $\|u(t) h-h u(t)\|$ $<\sqrt[4]{\delta}$. Выберем такой путь, соединяющий матрицу $d_{0}(\bar{u})$ с единицей так, чтобы этот путь не удалялся от унитарной группы более чем на $28\|h\|^{1 / 2} \sqrt[4]{\delta}$.

Итак, нами построен путь $u(t)$, соединяющий исходный оператор $u$ с единицей и такой, что $\operatorname{dist}(u(t), U)<28\|h\|^{1 / 2} \delta$ и $\|u(t) h-h u(t)\|<6 \sqrt[4]{\delta}$. Но тогда существует унитарный путь $u^{\prime}(t)$, такой, что $\left\|u^{\prime}(t)-u(t)\right\|<56\|h\|^{1 / 2} \sqrt[4]{\delta}$. При этом вдоль этого пути выполняется требуемая оценка

$$
\left\|u^{\prime}(t) h-h u^{\prime}(t)\right\| \leqslant\|u(t) h-h u(t)\|+2\|h\|\left\|u^{\prime}(t)-u(t)\right\|<C \sqrt[4]{\delta} .
$$

ЗАмечАниЕ. Тем же способом можно доказать аналогичный результат в случае, когда вместо самосопряженного оператора $h$ рассматривается унитарный оператор $v$, имеющий в спектре лакуну фиксированной длины.

Автор благодарен А. С. Мищенко и Е. В. Троицкому за полезные обсуждения.

\section{ЛитератУРА}

1. Brown L. G., Pedersen G. K. J. Funct. Anal., 99, 131-149 (1991). 2. Exel R., Loring T. A. J. Funct. Anal., 95, 364-376 (1991). 3. Rieffel M. A. J. Operator Theory, 17, 237-254 (1987). 4. Voiculescu D. Acta Sci. Math. (Szeged), 45, 429-431 (1983).

Московский государственный университет

Поступило в редакцию 7 мая 1996 г.

УдК 517.164.152

\section{Критические точки произвольных

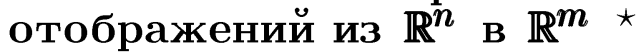

(c) 1997. М. А. ПАнков, Е. А. ПолУлях

1. Введение. В работах М. Гусмана $[1,2]$ можно найти следующее определение критической точки произвольного отображения $f: \mathbb{R}^{n} \rightarrow \mathbb{R}^{n}$. Точка $x \in \mathbb{R}^{n}$ называется критической (в смысле Гусмана) точкой отображения $f$, если существует система $n$-мерных кубов $\left\{K_{i}\right\}_{i=1}^{\infty}$ с центрами в точке $x$, диаметры которых стремятся к 0 при $i \rightarrow \infty$, и таких, что

$$
\lim _{i \rightarrow \infty} \frac{\operatorname{mes} f\left(K_{i}\right)}{\operatorname{mes} K_{i}}=0 .
$$

Здесь и далее mes - внешняя мера Лебега (напомним, что внешняя мера определена для произвольного подмножества пространства $\left.\mathbb{R}^{n}\right)$. Несложно показать корректность данного определения, т. е. если $f \in C^{1}\left(\mathbb{R}^{n}, \mathbb{R}^{n}\right)$, то точка $x$ принадлежит $\Sigma(f)$ тогда и только тогда, когда она является критической точкой в

* Работа выполнена при финансовой поддержке Международного научного фонда Дж. Copoca (грант U6F200). 